\title{
Genome-Wide Identification and Expression Profiling Analysis of Wnt Family Genes Affecting Adipocyte Differentiation in Cattle
}

\author{
Cuili Pan \\ Ningxia University \\ Shuzhe Wang \\ Ningxia University \\ Chaoyun Yang \\ Ningxia University \\ Chunli Hu \\ Ningxia University \\ Hui Sheng \\ Ningxia University \\ Xiaoshu Xue \\ Ningxia University \\ Honghong $\mathrm{Hu}$ \\ Ningxia University \\ Zhaoxiong Lei \\ Ningxia University \\ Mengli Yang \\ Ningxia University \\ Yun Ma ( $\nabla$ mayun_666@126.com ) \\ Ningxia University
}

\section{Research Article}

Keywords: Wnt, Cattle, adipocytes differentiation, expression profiling, collinearity analysis

Posted Date: July 13th, 2021

DOI: https://doi.org/10.21203/rs.3.rs-695671/v1

License: (c) (1) This work is licensed under a Creative Commons Attribution 4.0 International License. Read Full License

Version of Record: A version of this preprint was published at Scientific Reports on January 11th, 2022. See the published version at https://doi.org/10.1038/s41598-021-04468-1. 


\section{Abstract}

Wnt is a family of conserved glycoproteins that functions in a variety of crucial biological processes including tissue regeneration, animal development, and cell proliferation and differentiation. For its functional diversity and importance, Wnt gene family has gained considerable research interest in a variety of species. However, comprehensive identification and analysis of Wnt genes in Bovinae is lacking. In this study, we identified the repertoire of Wnt genes in cattle and seven other species of Bovinae and obtained 19 Wnt genes. Protein properties of these Wnt genes were also described. Phylogenetic analysis showed that the 149 Wnt proteins in Bovinae, together with 38 Wnt proteins from human and mouse, were clustered into 12 major clades. The Wnt genes belonging to the same subfamilies shared similar protein motif compositions and exonintron patterns. Chromosomal distribution and collinearity analysis of Wnt genes among cattle and five species of Bovinae revealed that this gene family was conservative in evolution. RNA-seq data analysis indicated that Wnt genes exhibited tissuespecific expression patterns in cattle. qPCR analysis of Wnt gene family showed that each gene had a unique expression pattern during bovine adipocytes differentiation. And the comprehensive analysis indicated that Wnt2B may regulate adipose differentiation through activation of FZD5, which is worthy of further study. Our study presents the first genome-wide study of Wnt gene family in Bovinae, and lay the foundation for further functional characterization of the Wnt family in bovine adipocytes differentiation.

\section{Introduction}

Wnt proteins, the initiators of the Wnt signaling pathway, comprise a large family of secreted glycoproteins that rich in cysteine[41]. The transduction of Wnt signaling mainly includes three pathways: the canonical Wnt (Wnt/ $\beta$-catenin) signaling pathway, the non-canonical Wnt/Ca2+ pathway, and the non-canonical planar cell polarity (PCP) pathway. The three patterns all require to bind to transmembrane receptor Frizzled to regulate intracellular responses. In the canonical Wnt pathway, the activated transmembrane receptor Frizzled ( Fzd) lead to a stable accumulation of $\beta$-catenin in the cytoplasm and translocation to the karyon. Then $\beta$-catenin bind to the transcription factor T-cell factor/lymphoid enhancing factor (LEF1/TCF) family, and activate the transcription of target genes to regulate embryo development, tissue regeneration, and cell proliferation and differentiation[24, 36, 56, 64]. In the non-canonical Wnt/Ca2+ pathway, the activated receptor Fzd (mainly Fzd2), results in intracellular $\mathrm{Ca} 2+$ release and irritates $\mathrm{Ca} 2+-$ calmodulin-dependent protein kinase II (CamKII) calnexin (CaN), and protein kinase $C(P K C)$, thus regulating cell adhesion and gene transcription[14]. In the non-canonical Wnt/PCP pathway, when the receptor Fzd is stimulated by Wnt proteins (e.g., Wnt5a and Wnt11), signals transmission are through Disheveled (Dvl) to trimeric G proteins. This is followed by activating downstream target genes Rho-associated kinase (Rock) and Jun N-terminal serine/threonine kinase (JNK), thereby regulating cytoskeletal actin and cell polarity[2,63].

Furthermore, studies have shown that Wnt signaling plays an important role in maintaining the undifferentiated state of precursor adipocytes and inhibiting adipogenesis. In 3T3-L1 preadipocytes, Wnt1 ectopic expression stabilizes $\beta$-catenin, thereby activating TCF-dependent gene transcription and blocking adipogenesis[7, 52]. Glucagon Like Peptide-1 (GLP-1) promotes adipogenesis through the up-regulation of the Wnt4 and $\beta$-catenin in the canonical Wnt signaling pathway[38]. KDM5A interacts with C/EBP $\beta$ and cooperatively inhibits the transcription of $W n t 6$, leading to the inhibition of Wnt/ $\beta$-catenin pathway and promotion of 3T3-L1 preadipocyte differentiation[22]. In human bone marrow stromal (mesenchymal) stem cell (hMSC), the treatment with Wnt3A activates Wnt/ $\beta$-catenin signaling pathway, thus decreasing in adipogenesis and increasing in osteogenesis[50, 51]. An activation of Wnt10B activates the Wnt signaling cascade and prevents the 3T3-L1 preadipocytes normal differentiation by inhibiting expression of $C / E B P a$ and $P P A R \gamma[23,26,52]$. In porcine adipose-derived mesenchymal stem cells (AMSCs), Wnt3A inhibits the potential of adipogenic differentiation and alters the cell fate from adipocytes to osteoblasts through Wnt/ $\beta$-catenin signaling pathway[34]. In murine embryonic mesenchymal cell line C3H10T1/2, Wnt3-Fz1 chimera is an inhibitor of differentiation into the adipocyte lineage and a potent activator of differentiation into osteoblasts[8]. It has been reported Wnt gene family also functions via non-canonical Wnt signaling pathway in addition to its role as a canonical Wnt ligand. In hMSC, an inhibition of Wnt3A can suppress the non-canonical Wnt/JNK pathway and enhance adipocyte differentiation whereas its activation enhance osteoblast differentiation[51]. In 3T3-L1 preadipocytes, Wnt4 and Wnt5A positively regulate adipogenesis at the initial stage of the differentiation process by activation of PKC and calcium/calmodulindependent kinase II[44]. Wnt5B partially represses canonical Wnt/ $\beta$-catenin signaling pathway 
and enhances adipogenesis[27]. These findings stimulated our interest and guided us to explore the function of Wnt gene family on bovine adipocyte differentiation.

So far, the Wnt family has been extensively studied in some species (Drosophila melanogaster, Tribolium castaneum, Acyrthosiphon pisum, Anopheles gambiae, and Apis mellifera, etc. ) $[5,11,15,39,40,43]$. However, there was limited understanding on their expression patterns and regulatory mechanisms in bovine adipocytes differentiation. To investigate the evolution of Wnt family genes in Bovinae and elucidate the function in bovine adipocytes differentiation, we carried out a genome-wide identification and evolutionary analysis of Wnt gene family in eight species of Bovinae. And the expression profiles in different tissues and stages during adipocytes differentiation were also analyzed in cattle based on transcriptome data and qPCR, respectively. Our study provides a basis for understanding the distribution of Wnt genes in Bovinae and is beneficial to further study of potential function of Wnt genes in bovine adipocytes differentiation.

\section{Results}

\section{The Wnt Gene Repertoire in Bovinae}

To identify the Wnt family members, 45 verified Wnt amino acid sequences were used as the query. These belonged to cattle (Bos taurus, 7), human (Homo sapiens, 19) and mouse (Mus musculus, 19). We used query sequences for genome-wide detection of homologous sequences in Bos taurus, Bos indicus, Hybrid-Bos taurus, Hybrid-Bos Indicus, Bos grunniens, Bos mutus, Bubalus bubalis and Bison bison bison. In Bos taurus, 19 non-redundant Wnt protein sequences were identified that included Wnt1-4, Wnt7A-10A, Wnt7B-10B, Wnt2B, Wnt3A, Wnt5A, Wnt5B, Wnt6, Wnt11, and Wnt16 (Table 1). Wnt family proteins were also recognized in Bos indicus (17), Hybrid-Bos taurus (19), Hybrid-Bos Indicus (19), Bos grunniens (19), Bos mutus (19), Bubalus bubalis (19) and Bison bison bison (18). (Supplementary info File 1 and 2). Two unannotated genes of the Wnt family, ENSBMUG00000022627 and ENSBMUG00000022624, were identified in Bos mutus. Further analysis revealed that they both have the WNT conserved domain, whereas ENSBMUG00000022627 has an incomplete N terminal and ENSBMUG00000022624 has an incomplete $\mathrm{C}$ terminal. They showed the highest sequence similarity and a collinearity relationship with Wnt7B, and were named Wnt7B1 and Wnt7B2 preliminary (Supplementary info File 3).

The amino acid lengths of the 19 bovine Wnt proteins ranged from 333 (Wnt8B) to 585 (Wnt4), whereas molecular weight (Mw) ranged from 36.61 to $63.17 \mathrm{kDa}$, consistent with protein length. Except Wnt3, the rest Wnt family proteins had the isoelectric points ( $\mathrm{pl}$ ) that higher than 8.0, as they contained more basic amino acids than acidic amino acids. Wnt3 was neutral, with a pl of 7.73. All 19 Wnt proteins contained the WNT conserved domain (Supplementary info File 4).

\section{Structural features of bovine Wnt family members}

In order to learn about the structural characteristics of bovine Wnt proteins and genes, we projected the conserved motifs and gene structures based on their phylogenetic relationships (Fig. 1). According to the evolutionary clades, the 19 bovine Wnt family menbers clustered into six main subfamilies ( $(-\mathbb{Z})$. All the Wnt family proteins shared six conserved domains termed motifs 1,2 , 4, 5, 6, and 7 formed by 50, 49, 41, 29, 28 and 9 amino acids, respectively (Supplementary info File 5). Wnt2, Wnt5A and Wnt5B of the first subfamily and Wnt3 of the third subfamily have all ten motifs. Wnt2B, Wnt3A and Wnt4A have nine motifs, since Wnt2B lacks 9, Wnt3A and Wnt4A lacks 10. Wnt7A, Wnt7B, Wnt10A, Wnt10B and Wnt1 (lacking 9 and 10) and Wnt1 (lacking 8 and 10) have eight motifs. Wnt8A, Wnt8B, Wnt11 and Wnt16 comprise seven motifs lacking 8,9 and 10. Wnt9A and Wnt9B comprise six motifs lacking 3, 8, 9 and 10.

Introns, coding sequences (CDS) and untranslated regions (UTR) were variable among the Wnt gene family. For instance, the length of Wnt genes ranged from 3,084 nt (Wnt7) to 64,231 nt (Wnt7A), mainly due to intron variation. The number of CDS varied from 3 to 6, and the length and layout of the noncoding areas, $3^{\prime} \mathrm{UTR}$ and 5'UTR, were also variable. Despite this variability in CDS, introns and UTRs, the Wnt members in the same evolutionary subfamily tend to possess similar patterns in gene structures and conserved motifs.

Phylogenetic relationship of Wnt proteins in different organisms

Page $3 / 21$ 
Phylogenetic analysis can provide a reference for understanding functional diversification of the Wnt family in Bovinae. So, phylogenetic analysis was conducted, which included eight Bovinae species (Bos taurus, Bos indicus, Hybrid-Bos taurus, HybridBos Indicus, Bos grunniens, Bubalus bubalis, Bos mutus, and Bison bison bison) and Homo sapiens and Mus musculus. Since Wnt proteins in model organisms (human and mouse) have been studied extensively, they were also included in this study. Finally, 186 amino acid sequences from these ten species were aligned to generate a nonrooted Neighbor-Joining (NJ) tree (Fig. 2). It revealed that Wnt family proteins could be subdivided into 12 proposed subfamilies, including Wnt1-11 and Wnt16

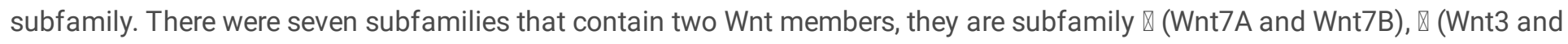
Wnt3A), $\otimes($ Wnt2 and Wnt2B), $\otimes(W n t 5 A$ and Wnt5B), $\otimes(W n t 10 A$ and Wnt10B), $\otimes($ Wnt9A and Wnt9B), $\otimes(W n t 8 A$ and Wnt8B), respectively.

\section{Chromosomal distribution and collinearity analysis of Wnt genes}

Wnt genes were mapped on nine chromosomes in organisms of Bovinae (Fig. 3). The bovine Wnt genes showed a similar distribution with the other five species. However, the order of Wnt1 (30832513-30835596 bp) and Wnt10B (30841487-30847512 bp) in Chr 5 and Wnt3A (3035810-3087361 bp) and Wnt9A (3155441-3163239 Mb) in Chr 7 of Bos taurus was reversed from that in Bos grunniens. In addition, compared with Bos taurus, Wnt9B and Wnt16 were lacking in Bos Indicus.

Genome collinearity analysis revealed a satisfactory corresponding relationship between chromosomes of Bos taurus and Hybrid-Bos Indicus, Hybrid-Bos taurus, Bos indicus and Bos grunniens, respectively (Fig. 4a). Although the chromosome number is different between cattle $(2 N=60)$ and buffalo $(2 N=50)$, there is also large chromosome homology between these two species. Also, collinearity modules explain the difference in the position of Wnt gene family between cattle and the other five species in Bovinae. For instance, the position variation of Wnt2B, Wnt11, Wnt1 and Wnt10B between Bos taurus and Bos grunniens may be caused by complex intra-chromosomal translocation events (Fig. 4b), whereas Wnt3 and Wnt9B are distributed on different chromosomes between cattle and buffalo (bovine Chr 19 and buffalo Chr 3), which may be caused by inter-chromosomal rupture or fusion during the evolutionary process (Supplementary info File 6).

\section{Expression analysis of Wnt genes in different tissue}

The functionally related genes tend to show a co-expression patterns and these genes often regulate biological processes collaboratively. To explore the expression patterns of the Wnt gene family during adipogenic differentiation, we investigated their expression levels in 163 samples of 60 tissue types. The Wnt genes along with other 13 closely related genes can be classified into four groups ( $\nabla$ to $\nabla$ ) (Fig. 5a) according to their differential expression patterns in diverse tissues. Accordingly, the 60 bovine tissue types also clustered into four main clades (a-d) based on the expression patterns of all the 31 genes including Wnt family. The members of Wnt family and its receptors, FZD gene family, displayed similar and overlapping expression patterns in the 60 tissues, suggesting their broad and coordinated regulatory role in life activities.

$P P A R Y$, a marker gene for adipocyte differentiation, showed high expression in Group a which included omental, intramuscula, subcutaneous and mammary gland fats. And CTNNB1, FZD1, FZD5, FZD6 and Wnt2B clustered and showed the most similar expression pattern with PPARY. Further analysis of the five different fat tissues revealed that CTNNB1, a core gene of Wnt signaling pathway, showed high expression and similar expression patterns with PPARY (Fig. 5b).

\section{Isolation and induced differentiation of bovine primary adipocytes}

To explore the expression patterns of the Wnt gene family during adipocyte differentiation, primary adipocytes collected from subcutaneous adipose tissue of calves were induced. The results of oil red 0 staining showed that lipid droplet gathered together in adipocytes induced for 10 days compared to preadipocytes (Fig. 6a). Further analysis revealed that the absorbance of the differentiated adipocytes at $260 \mathrm{~nm}$ was significantly higher than that of the preadipocytes (Fig. 6b). Also, The adipogenic marker genes (PPARY, CEBP $3, F A B P 4$ and $L P L$ ) were up-regulated (Fig. 6c). These results indicate that an induced differentiation system of primary adipocytes was established successfully, which can be used in the subsequent gene expression analysis.

\section{Expression analysis of Wnt genes during adipocyte differentiation}


A qPCR analysis was conducted to detect the expression of Wnt genes and their receptors (FZD genes) at 0,2, 6, and 10 days during adipocytes differentiation (Fig. 7). The members of Wnt8B, Wnt11, Wnt16 and their receptors Fzd1, Fzd2, Fzd3, Fzd4, Fzd6 showed a relatively high expression in preadipocytes and then reduced with the process of induced differentiation, suggesting that they may collectively play a role in keeping adipocytes undifferentiated. The members of Wnt2, Wnt6, Wnt9B, Wnt10A and their receptors $F z d 9, F z d 10$ showed an up-regulated tendency, indicating that they may play a regulatory role during adipocyte differentiation. Furthermore, Wnt2B, Wnt4, Wnt8A and Fzd5, Fzd8 reached the lowest expression on the second day and displayed a similar overall trend of expression.

\section{Discussion}

\section{Structural features of bovine Wnt family proteins and genes}

The proteins' core motifs and domains determine their function and activity [57]. Gene families usually encode a group of proteins that possess similar active sites and play a regulatory role synergistically, and they tend to have similar conserved motifs[19]. There are six conserved amino acid sequences (Motifs 1, 2, 4, 5, 6 and 7) in all the 19 bovine Wnt family members, pointing to a common functional site. Four Wnt family members (Wnt2, Wnt3, Wnt5A, and Wnt5A) have all of the ten motifs, whereas the other 15 members lack of 1 to 4 motifs. It is likely that these four motifs (Motifs $3,8,9$ or 10) are not located at the core of the Wnt proteins domain.

As the introns and UTRs vary in length and layout, the CDSs distribution of Wnt genes is also variable. Whereas their amino acid sequences and motif patterns, especially in conserved motifs, were similar, and they all have the WNT conserved domain, which play essential roles in keeping their three-dimensional structure and binding function.

\section{Phylogenetic relationship of Wnt family proteins}

Phylogenetic analysis provides a credible way to explore the relationship between acid sequence similarity and function of proteins in the same family[54]. By focusing on the phylogenetic relationship of Wnt family proteins in previous studies of multicellular eukaryotes, it showed that they can divided into 13 subfamilies. For instance, a total of 11 (Zhikong scallop), 12 (Yesso scallop, Pacific oyster), 13 (Lingula anatine, Plathynereis dumerlii, Lottia gigantean, Crassostrea gigas, etc.) subfamilies were identified, respectively[39]. In Bovinae, Wnt family proteins were classified into 12 sunfamilies lacking of WntA. It is consistent with the result that vertebrates have reserved all subfamilies except WntA[21, 49]. Although the Wnt family is highly conserved in molecular function, large-scale analysis showed that several Wnt members have been lost in many species after the complete set of Wnt genes emerged in cnidarians[31,33]. Among the eight species in Bovinae, Wnt7B is missing in Bison, and Wnt9B and Wnt16 is missing in Bos indicus. The Wnt9 subfamily specific to Bilateria was also found missing in Chlamys farreri [39]. It remains unclear whether these genes were failed to identify due to genome assembly limitation or they have been lost during species evolution. In the phylogenetic relationship, two genes from distinct species located in the same clade were defined as orthologs[29]. The orthologous gene pairs among cattle and the other five bovine species were identified based on the homologous relationships. Results showed that the orthologous Wnt members first clustered in one clade, indicating that they were conserved among different species.

\section{Collinearity analysis of Wnts in Bovidae}

The gene duplication events including tandem duplication and segmental duplication can cause gene family expansion in genome evolution[32]. Chromosome distribution and collinearity analysis indicated that both tandem duplication and segmental duplication were the reasons for the expansion of Wnt family in Bovinae (Fig. 3-4). The members of the Wnt gene family distributed in nine chromosomes in the six selected species. Due to the different starting point of chromosome annotation among species, the arrangement of genes may be totally reversed. For instance, the order of Wnt6, Wnt10A and Wnt4 in Chr2, Wnt2 and Wnt16 in Chr4, and Wnt3A, Wnt9A and Wnt8A in Chr 7 was totally opposite between Bos taurus and Hybrid-Bos taurus. Besides, intra-chromosomal translocation and rearrangement during species evolution also lead to the changes in gene arrangement $[45,61]$. For example, the position of Wnt2B between Bos taurus and Bos grunniens altered due to the inversion of large segments within the chromosome (Fig. 4B). It lead to the positions where Wnt genes located change from collinear 
(conserved in the same order) to syntenic (not necessarily in the same order) between two species [37]. In addition, buffalo Chr 1-5 are collinear with bovine Chr 27 and Chr 1, Chr 23 and Chr 2, Chr 19 and Chr 8, Chr 5 and Chr 28, and Chr 16 and Chr 29 , respectively (Supplementary info File 6). It is speculated to be caused by inter-chromosomal rupture or fusion during the evolutionary process. In a word, genome-wide collinearity analysis of Wnts provided rich perspectives for studying the function and evolution of genes in Bovidae.

\section{Wnt genes affecting adipocyte differentiation}

Wnt family proteins can activate their receptors, Fzd family proteins, and regulate adipocyte differentiation through the canonical and/or non-canonical Wnt signaling pathway. As it's known to all, functionally related genes usually exhibit similar expression patterns and gene expression clustering analysis could group together genes of similar function efficiently[17]. Previous studies have shown that Wnt is selective in recognizing its Fzd receptors[58]. For instance, Wnt3 formed a chimera with Fzd1 to regulate the canonical Wnt signaling[8]. To dissect the expression pattern of this family, the expression of 19 Wnt members and $10 \mathrm{Fzd}$ members in 60 tissue types was analyzed. The overlapping in expression patterns may suggest their coordinated and selective regulatory role (Fig. 5). Group $\triangle(P P A R G, C T N N B 1$, FZD1, FZD5, FZD6 and WNT2B) were highly expressed in four fat tissues (omental, intramuscular, subcutaneous and mammary gland). Meanwhile, the studies in human showed that Wnt2B and FZD5 have physical interaction and co-expression relationship (Table 2). Furthermore, they displayed similar expression tendency during the differentiation of bovine adipocytes (Fig. 7). As PPARG is a marker gene for adipocyte differentiation and CTNNB1 is a core gene for canonical Wnt signaling pathway, it is hypothesized that WNT2B may bind to its receptor $F Z D 5$ and regulate adipogenic differentiation through canonical Wnt signaling pathway.

Adipogenic differentiation is a well-organized and complicated process regulated by various genes. Analysis of the interaction relationship of Wnt and Fzd is essential to explore their roles. An integrated network of the Wnt and Fzd gene family and their interacting genes was constructed by STRING (https://string-db.org/) and visualized by Cytoscape (Supplementary info File 7) [53]. In order to ensure the accuracy of the interaction network, only the evidences of literature mining and experimental verification are selected. Results showed there are extensive and complex direct or indirect relationships between Wnt and Fzd gene family. Since there are limited study on the interaction between Wnt gene and FZD gene to regulate Wnt signaling pathway in cattle, GeneMANIA (https://genemania.org/) was used to mine their relationship in human. Results displayed the bias of Wnt family members in recognizing their transmembrane receptors, Fzd family proteins (Table 2) $[9,58]$, which provided reference on the study of cattle. Overall, these results revealed that the Wnt genes interact with Fzd genes, activating the canonical and/or non-canonical Wnt signaling pathway, and collectively regulating adipocyte differentiation. It laid the foundation for further research on Wnt genes regulating adipocyte differentiation in cattle.

\section{Methods}

\section{Ethics statement}

Animal experiments were conducted in accordance with the requirement of the Regulations for the Administration of Affairs Concerning Experimental Animals (Ministry of Science and Technology, China, 2004). It is authorized by the Animal Ethics Committee of Ningxia University (permit number NXUC20200521). The calf used in the experiment was electric shocked before released and the primary adipocytes was isolated immediately, making all efforts to minimize its suffering. It also conforms to the requirements of American Veterinary Medical Association (AVMA) Guidelines. The reporting in the study follows the recommendations in the ARRIVE guidelines [46].

\section{Genome-wide identification of Wnt genes}

The genome and annotation of Bos taurus (ARS-UCD1.2.101 assembly), Hybrid-Bos taurus (Bos indicus $\times$ Bos taurus, UOA_Angus_1.101 assembly), Hybrid-Bos Indicus (Bos indicus×Bos taurus, UOA_Brahman_1.101 assembly), Bos grunniens (LU_Bosgru_v3.0.101 assembly), Bos mutus (BosGru_v2.0.101 assembly), Bison bison bison (Bison_UMD1.0.101 assembly), Homo sapiens (GRCh38.101 assembly) and Mus musculus (GRCm38.101 assembly) are downloaded from Ensembl database (http://asia.ensembl.org/index.html), Bos indicus (GCF_000247795.1 assembly) and Bubalus bubalis (ASM312139v1 
assembly) are downloaded from NCBI database (https://www.ncbi.nlm.nih.gov/). To identify all the possible Wnt genes in Bovinae, both Basic Local Alignment Search Tool (BLAST) and Hidden Markov Model (HMM) search were performed[35]. A total of 45 reviewed Wnts sequences of bovine (7), human (19) and mouse (19) obtained from the UniProt database (https://www.uniprot.org/) were used as seeds to query potential Wnt genes via BLASTP with a threshold of e-value $=10^{-5}$. The HMM of Wnt (PF00110) was downloaded from Pfam (https://pfam.xfam.org/)[18] and HMMER 3.3.1 (http://hmmer.org/)[48] was conducted to construct HMM profiles in Bovidae to detect Wnt genes with the default setting. Candidate sequences were manually checked to confirm the Wnt homology.

\section{Analysis of Wnt Gene Characteristics}

Protein properties of identified Wnt genes, including protein length, molecular weights, and isoelectric points, were estimated using ExPASy (https://web.expasy.org/protparam/)[4]. The conserved motifs were detected in MEME 5.0[6]. The minimum and maximum number of amino acids in each motif was 6 and 50, respectively. Exon-intron structures and motif patterns of Wnt family were displayed using TBtools software [12]. NCBI-CDD [42] was used to identify the conservative domain compositions of 19 Wnt proteins, and the results were visualized by the TBtools software [12]. Multiple sequence alignments of Wnts and a phylogenetic Neighbor-Joining tree was constructed using the MEGA 7.0 software [30].

\section{Phylogenetic Tree Construction, Chromosomal distribution, and Collinearity analysis}

To investigate the phylogenetic relationship of Wnt genes, the protein sequences were aligned using ClustalW. And a NeighborJoining tree was constructed in MEGA 7.0[30] using the following parameters: bootstrap method (1000 replicates), Poisson model, and complete deletion. FigTree software (version 1.4.3) was used to adjust and beautify the evolutionary tree.

Chromosomal locations of Wnt genes in cattle and other five species in Bovinae were obtained from general feature format (GFF3) files. Gene Location Visualize from GFF [12]was used to map the distribution of Wnt genes. Collinearity analysis of ortholog genes between Bos taurus and the other five bovine species was performed using the MCScanX toolkit[59].

Subsequently, genome collinearity results and orthologous Wnts were visualized by Dual Systeny Plot for MCscanX [12].

Expression Profiles of Wnt Genes in RNA-Seq

RNA-Seq data of 163 tissue samples were collected from the Ruminant Genome Database

(http://animal.nwsuaf.edu.cn/code/index.php/Ruminantia) [13] and all the raw data was deposited in the National Center for Biotechnology Information (NCBI) Sequence Read Archive (Supplementary info File 8). The sequencing quality was checked using FastQC[62]. Quality control of raw sequence data was performed using the trimmomatic-0.36 [10]. Clean reads were then mapped to the Bos taurus genome reference using STAR [16] and Hisat2 [28]. FPKMs (Fragments Per Kilobase per Million mapped reads) of the genes in each sample were computed by Ballgown (Version 2.2.0) [20,47]. The heat map was constructed using theTBtools software [12].

\section{Isolation, culture and induction differentiation of bovine primary adipocytes}

Primary adipocytes were isolated and cultured from subcutaneous adipose tissue of the calf in the Zerui ecological breeding farm. The primary adipocytes were isolated and cultivated by Type $\varangle$ collagenase digestion method. The induction of primary adipocytes differentiation [25] and oil red 0 staining [60] were performed as previous described. The substance extracted from adipocytes induced at day 0 day and 10 day were also measured at $510 \mathrm{~nm}$ absorbance with isopropanol as control.

\section{RNA extraction and quantitative RT-PCR (qPCR)}

The primers of the Wnt genes were designed using Primer Premier 5.0 software (Supplementary info File 9). Total RNA was extracted by the phenol-chloroform method using TRIzol (9109, Takara) and samples with an OD260/OD280 absorbance ratio between 1.8 and 2.0 were used in the subsequent experiments. Then, a total of $1000 \mathrm{ng}$ RNA was reverse transcribed using random primers with Moloney murine leukemia virus reverse transcriptase (Takara Bio, Kyoto, Japan). Realtime PCR was carried out in a CFX96 Touch Real-Time PCR Detection System (Bio-Rad, Hercules, CA, USA) with SYBR Green Master Mix (Takara Bio, Kyoto, Japan). The qPCR reaction procedure was 40 cycles of pre-denaturation for $3 \min \left(95^{\circ} \mathrm{C}\right)$, denaturation for $10 \mathrm{~s}\left(95^{\circ} \mathrm{C}\right)$, 
annealing for $20 \mathrm{~s}\left(60^{\circ} \mathrm{C}\right)$, extension for $30 \mathrm{~s}\left(72^{\circ} \mathrm{C}\right)$. Relative expression was calculated using the $2-\Delta \Delta \mathrm{Ct} \operatorname{method}[1,3]$ with $\beta$ actin as the reference. Three replicates were performed for each test. Statistical significance determined using Graphpad Prism 7.0 software.

\section{Declarations}

\section{Acknowledgments}

We would like to thank all the participants.

Authors' contributions $₫$ CLP and SZW made the same contribution to the work. Conceived and designed the research:YM and CLP, Analyzed the data and conducted the experiment: CLP, SZW and CLH, Wrote the paper: CLP, Modified manuscript: YM, CYY, HS, XSX, HHH, ZXL and MLY. All authors read and approved the final manuscript.

\section{Competing interests}

The authors declare no competing interests.

\section{Availability of data and materials}

All data generated or analyzed during this study are included in this published article and its supplementary information files.

\section{Consent for publication}

Not applicable.

\section{Ethics approval and consent to participate}

The Animal Ethics Committees of Ningxia University approved the experimental design and animal sample collection (permit number NXUC20200521). We also obtained the informed consent to participate from the owners and the Ethics Committees of Zerui ecological breeding farm. All the animal experiments were conducted according to the guidelines of the Regulations for the Administration of Affairs Concerning Experimental Animals (Ministry of Science and Technology, China, 2004). The reporting in the study follows the recommendations in the ARRIVE guidelines.

Funding $\$ This study was funded by the National Natural Science Foundation of China (32072720 and 31672403), the Leading Talents Fund in Science and Technology Innovation in Henan Province (No. 194200510022), the Key Research and Talent Introduction Project of Ningxia Hui Autonomous Region (2019YCZX0068) and the Science and Technology Innovation Team Projects of Ningxia Hui Autonomous Region (03010360052). The funding bodies played no role in the design of the study, collection, analysis, and interpretation of data and writing the manuscript.

\section{References}

1. Adnan, M., Morton, G. \& Hadi, S. Analysis of rpoS and bolA Gene Expression Under Various Stress-Induced Environments in Planktonic and Biofilm Phase Using 2(-DeltaDeltaCT) Method. MOL CELL BIOCHEM. 357, 275-282 (2011).

2. Andre, P., Song, H., Kim, W., Kispert, A. \& Yang, Y. Wnt5a and Wnt11 Regulate Mammalian Anterior-Posterior Axis Elongation. DEVELOPMENT. 142, 1516-1527 (2015).

3. Arocho, A., Chen, B., Ladanyi, M. \& Pan, Q. Validation of the 2-DeltaDeltaCt Calculation as an Alternate Method of Data Analysis for Quantitative PCR of BCR-ABL P210 Transcripts. Diagn Mol Pathol. 15, 56-61 (2006).

4. Artimo, P. et al. ExPASy: SIB Bioinformatics Resource Portal. NUCLEIC ACIDS RES. 40, W597-W603 (2012).

5. Bai, Y., Nie, H., Wang, Z. \& Yan, X. Genome-Wide Identification and Transcriptome-Based Expression Profiling of Wnt Gene Family in Ruditapes Philippinarum. Comp Biochem Physiol Part D Genomics Proteomics. 35, 100709 (2020).

6. Bailey, T. L., Johnson, J., Grant, C. E. \& Noble, W. S. The MEME Suite. NUCLEIC ACIDS RES. 43, W39-W49 (2015). 
7. Bennett, C. N. et al. Regulation of Wnt Signaling During Adipogenesis. J BIOL CHEM. 277, 30998-31004 (2002).

8. Bhat, R. A., Stauffer, B., Della, P. A. \& Bodine, P. V. Wnt3-Frizzled 1 Chimera as a Model to Study Canonical Wnt Signaling. $J$ CELL BIOCHEM. 109, 876-884 (2010).

9. Bhat, R. A., Stauffer, B., Della, P. A. \& Bodine, P. V. Wnt3-Frizzled 1 Chimera as a Model to Study Canonical Wnt Signaling. J CELL BIOCHEM. 109, 876-884 (2010).

10. Bolger, A. M., Lohse, M. \& Usadel, B. Trimmomatic: A Flexible Trimmer for Illumina Sequence Data. BIOINFORMATICS. 30 , 2114-2120 (2014).

11. Bolognesi, R. et al. Tribolium Wnts: Evidence for a Larger Repertoire in Insects with Overlapping Expression Patterns that Suggest Multiple Redundant Functions in Embryogenesis. DEV GENES EVOL. 218, 193-202 (2008).

12. Chen, C. et al. TBtools: An Integrative Toolkit Developed for Interactive Analyses of Big Biological Data. MOL PLANT. 13, 1194-1202 (2020).

13. Chen, L. et al. Large-Scale Ruminant Genome Sequencing Provides Insights Into their Evolution and Distinct Traits. SCIENCE. 364, (2019).

14. De A. Wnt/Ca2+ Signaling Pathway: A Brief Overview. Acta Biochim Biophys Sin (Shanghai). 43, 745-756 (2011).

15. Ding, X. et al. Genome-Wide Identification and Expression Profiling of Wnt Family Genes in the Silkworm, Bombyx mori. INT J MOL SCI. 20, (2019).

16. Dobin, A. \& Gingeras, T. R. Mapping RNA-seq Reads with STAR. Curr Protoc Bioinformatics. 51, 11-14 (2015).

17. Eisen, M. B., Spellman, P. T., Brown, P. O. \& Botstein, D. Cluster Analysis and Display of Genome-Wide Expression Patterns. Proc Natl Acad Sci U S A. 95, 14863-14868 (1998).

18. El-Gebali, S. et al. The Pfam Protein Families Database in 2019. NUCLEIC ACIDS RES. 47, D427-D432 (2019).

19. Esposito, D., Koliopoulos, M. G. \& Rittinger, K. Structural Determinants of TRIM Protein Function. Biochem Soc Trans. 45, 183-191 (2017).

20. Frazee, A. C. et al. Ballgown Bridges the Gap Between Transcriptome Assembly and Expression Analysis. NAT BIOTECHNOL. 33, 243-246 (2015).

21. Garriock, R. J., Warkman, A. S., Meadows, S. M., D'Agostino, S. \& Krieg, P. A. Census of Vertebrate Wnt Genes: Isolation and Developmental Expression of Xenopus Wnt2, Wnt3, Wnt9a, Wnt9b, Wnt10a, and Wnt16. Dev Dyn. 236, 1249-1258 (2007).

22. Guo, L., Guo, Y. Y., Li, B. Y., Peng, W. Q. \& Tang, Q. Q. Histone Demethylase KDM5A is Transactivated by the Transcription Factor C/EBPbeta and Promotes Preadipocyte Differentiation by Inhibiting Wnt/beta-catenin Signaling. J BIOL CHEM. 294, 9642-9654 (2019).

23. Hammarstedt, A., Isakson, P., Gustafson, B. \& Smith, U. Wnt-Signaling is Maintained and Adipogenesis Inhibited by TNFalpha but Not MCP-1 and Resistin. Biochem Biophys Res Commun. 357, 700-706 (2007).

24. Hu, H. H., Cao, G., Wu, X. Q., Vaziri, N. D. \& Zhao, Y. Y. Wnt Signaling Pathway in Aging-Related Tissue Fibrosis and Therapies. AGEING RES REV. 60, 101063 (2020).

25. Huang, J. et al. High-Throughput RNA Sequencing Reveals NDUFC2-AS IncRNA Promotes Adipogenic Differentiation in Chinese Buffalo (Bubalus bubalis L). Genes (Basel). 10, (2019).

26. Isakson, P., Hammarstedt, A., Gustafson, B. \& Smith, U. Impaired Preadipocyte Differentiation in Human Abdominal Obesity: Role of Wnt, Tumor Necrosis Factor-Alpha, and Inflammation. DIABETES. 58, 1550-1557 (2009).

27. Kanazawa, A. et al. Wnt5b Partially Inhibits Canonical Wnt/beta-catenin Signaling Pathway and Promotes Adipogenesis in 3T3-L1 Preadipocytes. Biochem Biophys Res Commun. 330, 505-510 (2005).

28. Kim, D., Paggi, J. M., Park, C., Bennett, C. \& Salzberg, S. L. Graph-Based Genome Alignment and Genotyping with HISAT2 and HISAT-genotype. NAT BIOTECHNOL. 37, 907-915 (2019).

29. Koonin, E. V. Orthologs, Paralogs, and Evolutionary Genomics. ANNU REV GENET. 39, 309-338 (2005).

30. Kumar, S., Stecher, G. \& Tamura, K. MEGA7: Molecular Evolutionary Genetics Analysis Version 7.0 for Bigger Datasets. MOL BIOL EVOL. 33, 1870-1874 (2016).

31. Kusserow, A. et al. Unexpected Complexity of the Wnt Gene Family in a Sea Anemone. NATURE. 433, 156-160 (2005).

Page $9 / 21$ 
32. Lawton-Rauh, A. Evolutionary Dynamics of Duplicated Genes in Plants. MOL PHYLOGENET EVOL. 29, $396-409$ (2003).

33. Lengfeld, T. et al. Multiple Wnts are Involved in Hydra Organizer Formation and Regeneration. DEV BIOL. 330, 186-199 (2009).

34. Li, H. X., Luo, X., Liu, R. X., Yang, Y. J. \& Yang, G. S. Roles of Wnt/beta-catenin Signaling in Adipogenic Differentiation Potential of Adipose-Derived Mesenchymal Stem Cells. MOL CELL ENDOCRINOL. 291, 116-124 (2008).

35. Li, Y., He, L., Li, J., Chen, J. \& Liu, C. Genome-Wide Identification, Characterization, and Expression Profiling of the Legume BZR Transcription Factor Gene Family. FRONT PLANT SCI. 9, 1332 (2018).

36. Lingappan, K. \& Savani, R. C. The Wnt Signaling Pathway and the Development of Bronchopulmonary Dysplasia. Am J Respir Crit Care Med. 201, 1174-1176 (2020).

37. Liu, J., Wang, Z., Li, J., Li, H. \& Yang, L. Genome-Wide Identification of Diacylglycerol Acyltransferases (DGAT) Family Genes Influencing Milk Production in Buffalo. BMC GENET. 21, 26 (2020).

38. Liu, R. et al. Glucagon Like Peptide-1 Promotes Adipocyte Differentiation via the Wnt4 Mediated Sequestering of BetaCatenin. PLOS ONE. 11, e160212 (2016).

39. Liu, S. et al. Genome-Wide Identification and Expression Profiling of the Wnt Gene Family in Three Bivalve Molluscs. Comp Biochem Physiol Part D Genomics Proteomics. 29, 299-307 (2019).

40. Liu, S. et al. Genome-Wide Identification and Expression Profiling of the Wnt Gene Family in Three Bivalve Molluscs. Comp Biochem Physiol Part D Genomics Proteomics. 29, 299-307 (2019).

41. Lorzadeh, S., Kohan, L., Ghavami, S. \& Azarpira, N. Autophagy and the Wnt Signaling Pathway: A Focus On Wnt/betacatenin Signaling. Biochim Biophys Acta Mol Cell Res. 1868, 118926 (2021).

42. Lu, S. et al. CDD/SPARCLE: The Conserved Domain Database in 2020. NUCLEIC ACIDS RES. 48, D265-D268 (2020).

43. Murat, S., Hopfen, C. \& McGregor, A. P. The Function and Evolution of Wnt Genes in Arthropods. ARTHROPOD STRUCT DEV. 39, 446-452 (2010).

44. Nishizuka, M., Koyanagi, A., Osada, S. \& Imagawa, M. Wnt4 and Wnt5a Promote Adipocyte Differentiation. FEBS LETT. 582, 3201-3205 (2008).

45. Pellestor, F. Chromoanagenesis: Cataclysms Behind Complex Chromosomal Rearrangements. MOL CYTOGENET. 12, 6 (2019).

46. Percie, D. S. N. et al. The ARRIVE Guidelines 2.0: Updated Guidelines for Reporting Animal Research. PLOS BIOL. 18, e3000410 (2020).

47. Pertea, M., Kim, D., Pertea, G. M., Leek, J. T. \& Salzberg, S. L. Transcript-Level Expression Analysis of RNA-seq Experiments with HISAT, StringTie and Ballgown. NAT PROTOC. 11, 1650-1667 (2016).

48. Potter, S. C. et al. HMMER Web Server: 2018 Update. NUCLEIC ACIDS RES. 46, W200-W204 (2018).

49. Prud'Homme, B., Lartillot, N., Balavoine, G., Adoutte, A. \& Vervoort, M. Phylogenetic Analysis of the Wnt Gene Family. Insights From Lophotrochozoan Members. CURR BIOL. 12, 1395 (2002).

50. Qiu, W. et al. Patients with High Bone Mass Phenotype Exhibit Enhanced Osteoblast Differentiation and Inhibition of Adipogenesis of Human Mesenchymal Stem Cells. J BONE MINER RES. 22, 1720-1731 (2007).

51. Qiu, W., Chen, L. \& Kassem, M. Activation of Non-Canonical Wnt/JNK Pathway by Wnt3a is Associated with Differentiation Fate Determination of Human Bone Marrow Stromal (Mesenchymal) Stem Cells. Biochem Biophys Res Commun. 413, 98104 (2011).

52. Ross, S. E. et al. Inhibition of Adipogenesis by Wnt Signaling. SCIENCE. 289, 950-953 (2000).

53. Shannon, P. et al. Cytoscape: A Software Environment for Integrated Models of Biomolecular Interaction Networks. GENOME RES. 13, 2498-2504 (2003).

54. Song, L., Wu, S. \& Tsang, A. Phylogenetic Analysis of Protein Family. Methods Mol Biol. 1775, 267-275 (2018).

55. Szklarczyk, D. et al. STRING V11: Protein-Protein Association Networks with Increased Coverage, Supporting Functional Discovery in Genome-Wide Experimental Datasets. NUCLEIC ACIDS RES. 47, D607-D613 (2019). 
56. Taciak, B., Pruszynska, I., Kiraga, L., Bialasek, M. \& Krol, M. Wnt Signaling Pathway in Development and Cancer. J PHYSIOL PHARMACOL. 69, (2018).

57. Vallbracht, M., Fuchs, W., Klupp, B. G. \& Mettenleiter, T. C. Functional Relevance of the Transmembrane Domain and Cytoplasmic Tail of the Pseudorabies Virus Glycoprotein H for Membrane Fusion. J VIROL. 92, (2018).

58. Wada, N. et al. Selective Modulation of Wnt Ligands and their Receptors in Adipose Tissue by Chronic Hyperadiponectinemia. PLOS ONE. 8, e67712 (2013).

59. Wang, Y. et al. MCScanX: A Toolkit for Detection and Evolutionary Analysis of Gene Synteny and Collinearity. NUCLEIC ACIDS RES. 40, e49 (2012).

60. Wang, Y., Goulart, R. A. \& Pantanowitz, L. Oil Red O Staining in Cytopathology. DIAGN CYTOPATHOL. 39, 272-273 (2011).

61. Weckselblatt, B. \& Rudd, M. K. Human Structural Variation: Mechanisms of Chromosome Rearrangements. TRENDS GENET. 31, 587-599 (2015).

62. Wingett, S. W. \& Andrews, S. FastQ Screen: A Tool for Multi-Genome Mapping and Quality Control. F1000Res. 7, 1338 (2018).

63. Yamanaka, H. et al. JNK Functions in the Non-Canonical Wnt Pathway to Regulate Convergent Extension Movements in Vertebrates. EMBO REP. 3, 69-75 (2002).

64. Yao, L. et al. The Effect of Regulating the Wnt Signaling Pathway On the Proliferation and Differentiation of Spermatogonial Stem Cells. Ann Transl Med. 8, 1003 (2020).

\section{Tables}

Table 1. Characteristics of Genome-wide identified Wnt family members in Bos taurus 


\begin{tabular}{|c|c|c|c|c|c|c|}
\hline $\begin{array}{l}\text { Gene } \\
\text { Name }\end{array}$ & Gene ID & Transcript ID & pl & $\mathrm{Mw} / \mathrm{kDa}$ & $\begin{array}{l}\text { Amino } \\
\text { acids }\end{array}$ & description \\
\hline WNT1 & ENSBTAG00000015364 & ENSBTAT00000020414 & 9.24 & 41.04 & 370 & Wnt family member 1 \\
\hline WNT2 & ENSBTAG00000008097 & ENSBTAT00000010650 & 9.21 & 40.64 & 360 & Wnt family member 2 \\
\hline WNT2B & ENSBTAG00000014291 & ENSBTAT00000018985 & 9.32 & 44.17 & 394 & $\begin{array}{l}\text { Wnt family member } \\
2 \mathrm{~B}\end{array}$ \\
\hline WNT3 & ENSBTAG00000016012 & ENSBTAT00000021312 & 7.73 & 39.78 & 355 & Wnt family member 3 \\
\hline WNT3A & ENSBTAG00000039397 & ENSBTAT00000056888 & 8.37 & 38.42 & 346 & $\begin{array}{l}\text { Wnt family member } \\
3 \mathrm{~A}\end{array}$ \\
\hline WNT4 & ENSBTAG00000051083 & ENSBTAT00000085976 & 9.11 & 63.17 & 585 & Wnt family member 4 \\
\hline WNT5A & ENSBTAG00000020221 & ENSBTAT00000026930 & 8.88 & 42.42 & 380 & $\begin{array}{l}\text { Wnt family member } \\
5 \mathrm{~A}\end{array}$ \\
\hline WNT5B & ENSBTAG00000054367 & ENSBTAT00000068978 & 8.99 & 40.05 & 358 & $\begin{array}{l}\text { Wnt family member } \\
5 B\end{array}$ \\
\hline WNT6 & ENSBTAG00000013990 & ENSBTAT00000018592 & 9.19 & 39.69 & 365 & Wnt family member 6 \\
\hline WNT7A & ENSBTAG00000001668 & ENSBTAT00000002188 & 9.05 & 38.90 & 349 & $\begin{array}{l}\text { Wnt family member } \\
7 A\end{array}$ \\
\hline WNT7B & ENSBTAG00000048365 & ENSBTAT00000083229 & 9.87 & 52.40 & 472 & $\begin{array}{l}\text { Wnt family member } \\
\text { 7B }\end{array}$ \\
\hline WNT8A & ENSBTAG00000005570 & ENSBTAT00000035270 & 8.25 & 39.06 & 351 & $\begin{array}{l}\text { Wnt family member } \\
8 \mathrm{~A}\end{array}$ \\
\hline WNT8B & ENSBTAG00000012914 & ENSBTAT00000017163 & 8.88 & 36.61 & 333 & $\begin{array}{l}\text { Wnt family member } \\
8 \mathrm{~B}\end{array}$ \\
\hline WNT9A & ENSBTAG00000020267 & ENSBTAT00000081055 & 8.88 & 39.85 & 362 & $\begin{array}{l}\text { Wnt family member } \\
9 A\end{array}$ \\
\hline WNT9B & ENSBTAG00000002664 & ENSBTAT00000086580 & 9.19 & 38.74 & 353 & $\begin{array}{l}\text { Wnt family member } \\
9 \mathrm{~B}\end{array}$ \\
\hline WNT10A & ENSBTAG00000009217 & ENSBTAT00000012148 & 9.36 & 46.34 & 417 & $\begin{array}{l}\text { Wnt family member } \\
10 \mathrm{~A}\end{array}$ \\
\hline WNT10B & ENSBTAG00000015347 & ENSBTAT00000020402 & 9.22 & 42.96 & 391 & $\begin{array}{l}\text { Wnt family member } \\
10 \mathrm{~B}\end{array}$ \\
\hline WNT11 & ENSBTAG00000010820 & ENSBTAT00000014355 & 9.14 & 39.24 & 354 & $\begin{array}{l}\text { Wnt family member } \\
11\end{array}$ \\
\hline WNT16 & ENSBTAG00000002940 & ENSBTAT00000003825 & 9.04 & 40.42 & 362 & $\begin{array}{l}\text { Wnt family member } \\
16\end{array}$ \\
\hline
\end{tabular}

Mw: molecular weight, pl: isoelectric point

Table 2. Interation relationship between Wnt and Fzd family genes in Human 
Gene Interaction types with Fzd family genes

Symbol

pl

$\mathrm{Mw} / \mathrm{kDa}$

Amino acids

description

\begin{tabular}{|c|c|c|}
\hline $\begin{array}{l}\text { Physical } \\
\text { interation }\end{array}$ & Co-expression & Predicted \\
\hline
\end{tabular}

\begin{tabular}{|c|c|c|c|c|c|}
\hline WNT1 & Fzd8 & - & Fzd1,Fzd3,Fzd5,Fzd8 & - & Fzd1,Fzd3,Fzd5,Fzd8 \\
\hline WNT2 & Fzd1,Fzd9 & Fzd1,Fzd2 & - & - & Fzd1,Fzd2,Fzd9 \\
\hline WNT2B & Fzd5 & Fzd5 & - & - & Fzd2,Fzd5 \\
\hline WNT3 & Fzd1,Fzd7 & - & - & - & Fzd1,Fzd2,Fzd7,Fzd8 \\
\hline WNT3A & Fzd1,Fzd2,Fzd8 & - & - & - & Fzd1,Fzd2,Fzd5,Fzd8 \\
\hline WNT4 & - & - & Fzd1,Fzd5,Fzd8 & - & Fzd1,Fzd2,Fzd5,Fzd8 \\
\hline WNT5A & Fzd1,Fzd2,Fzd5 & Fzd1,Fzd2,Fzd10 & - & Fzd5 & Fzd1,Fzd2,Fzd4,Fzd5,Fzd7,Fzd10 \\
\hline WNT5B & - & Fzd2 & - & - & Fzd2 \\
\hline WNT6 & - & Fzd2 & - & - & Fzd1,Fzd2 \\
\hline WNT7A & Fzd5,Fzd9 & Fzd9 & - & - & Fzd2,Fzd5,Fzd9 \\
\hline WNT7B & - & Fzd3 & Fzd1,Fzd5,Fzd8 & - & Fzd1,Fzd2,Fzd3,Fzd5,Fzd8,Fzd10 \\
\hline WNT8A & - & - & - & - & Fzd2 \\
\hline WNT8B & - & Fzd3 & - & - & Fzd2,Fzd3,Fzd8 \\
\hline WNT9A & - & - & - & - & Fzd2,Fzd8 \\
\hline WNT9B & - & - & - & - & Fzd2,Fzd8 \\
\hline WNT10A & - & Fzd9 & - & - & Fzd2,Fzd8,Fzd9 \\
\hline WNT10B & - & Fzd10 & - & - & Fzd2,Fzd8,Fzd10 \\
\hline WNT11 & - & Fzd4 & - & - & Fzd2,Fzd4 \\
\hline WNT16 & - & - & - & - & Fzd2 \\
\hline
\end{tabular}

'-' represents the absence of an interacting Fzd.

\section{Figures}




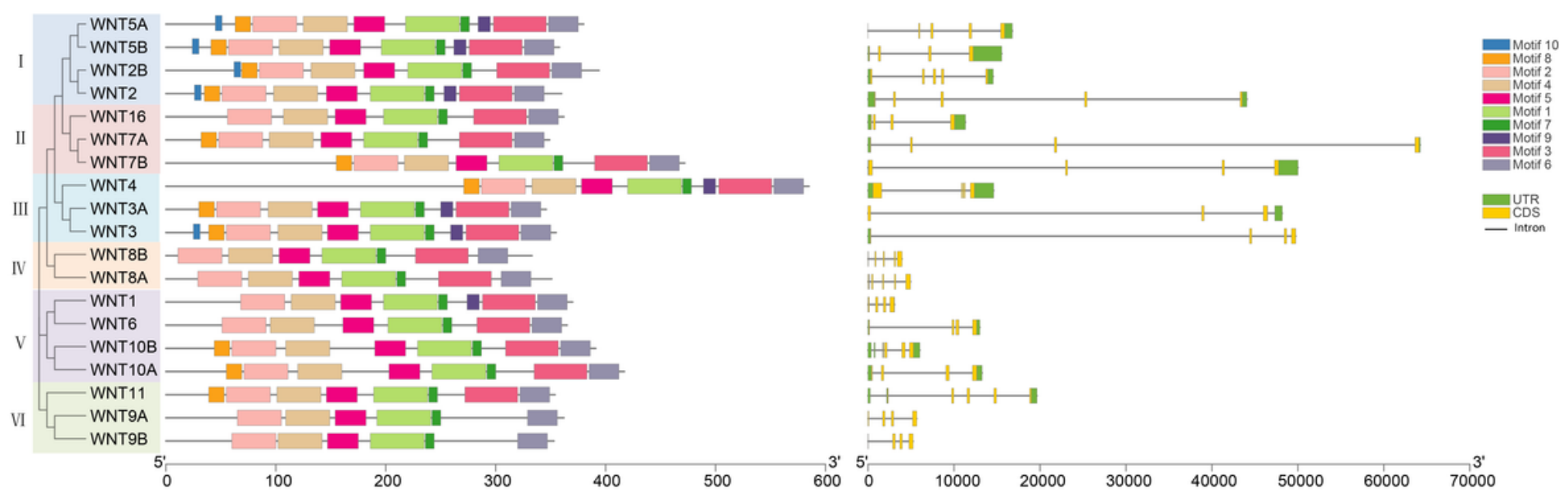

\section{Figure 1}

Characterization of the identified Wnt proteins and genes in Bos taurus. The phylogenetic tree (left) was constructed by the Neighbor Joining method. Motifs of amino acid sequences (middle), where conserved motifs are indicated as rectangles with different colors. Gene structure map (right), where yellow rectangle, black line and green rectangle represent CDS, intron and UTR, respectively. 


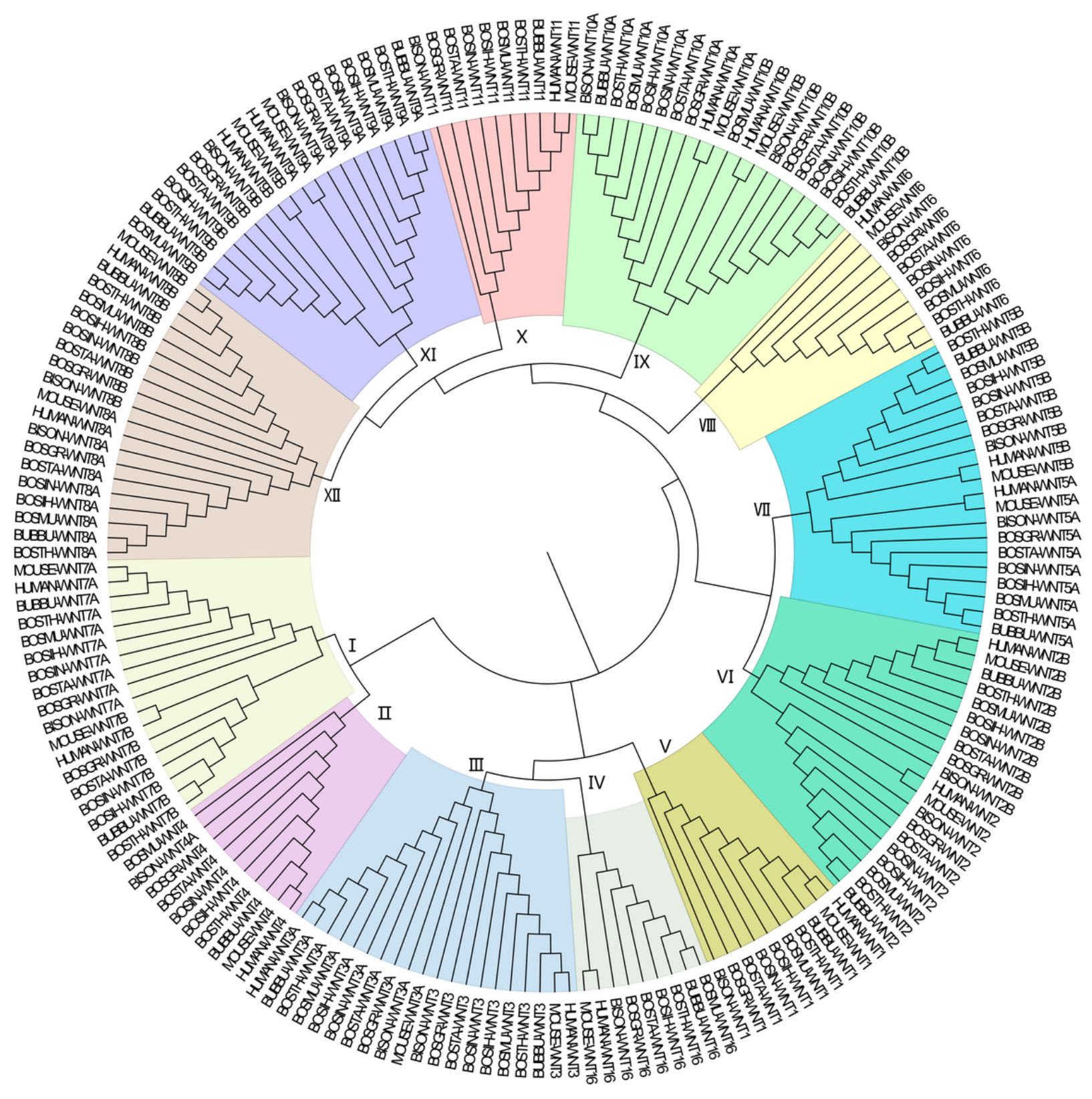

Figure 2

Phylogenetic Neighbor-Joining (NJ) tree of Wnt proteins from ten organisms. Identified Wnts in Bos taurus (BOSTA), Bos grunniens (BOSGR), Hybrid-Bos Indicus (BOSIH), Hybrid-Bos taurus (BOSTH), Bos mutus (BOSMU), Bison bison bison (BISOM), Bos indicus (BOSIN) and Bubalus bubalis (BUBBU) together with verified Wnts from Homo sapiens (HUMAN) and Mus musculus (MOUSE). Wnt proteins are grouped into 12 clusters $(\mathbb{Q}-\mathbb{Q})$ shown as different colors. 


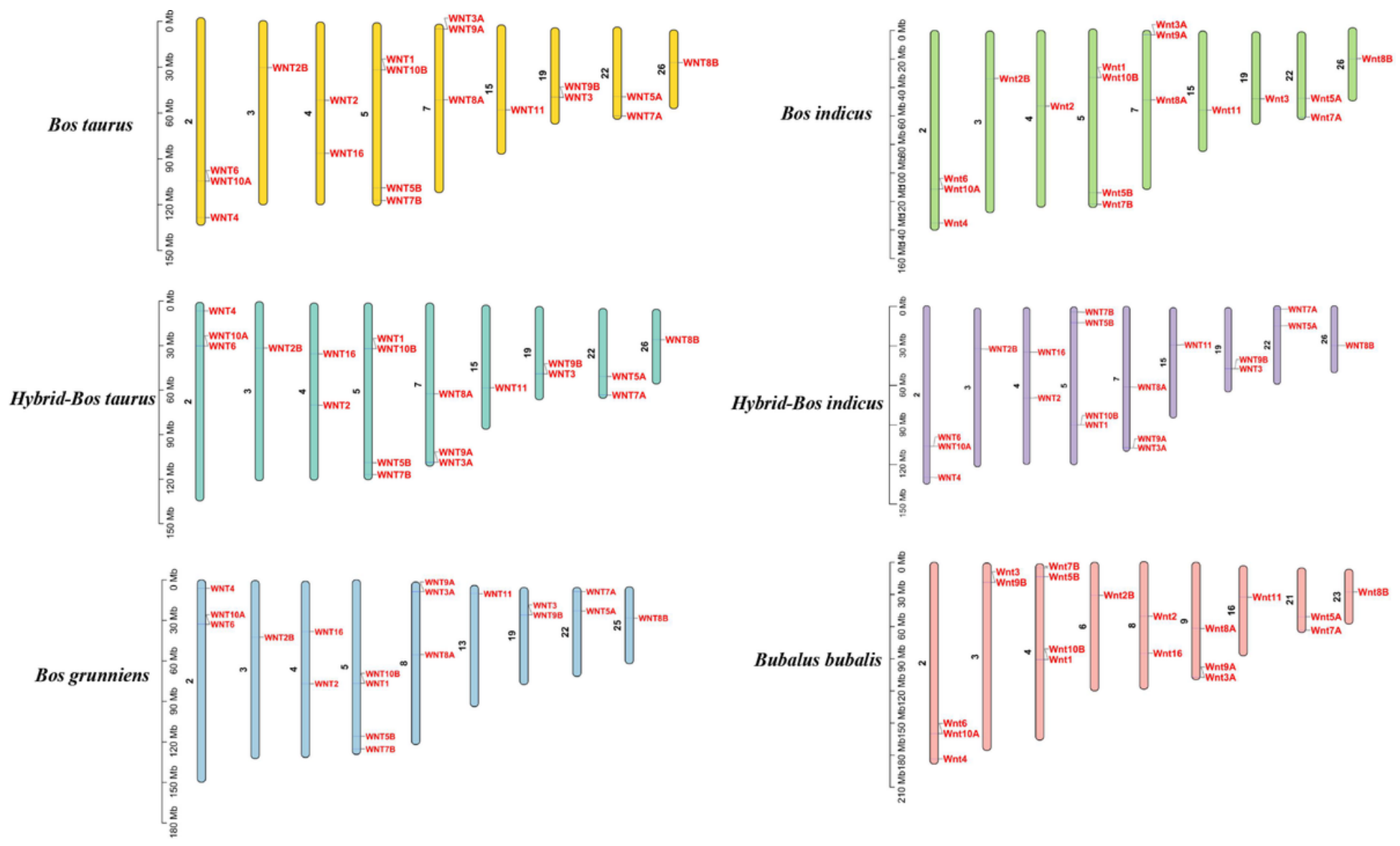

Figure 3

Chromosomal distribution of Wnt genes. The black font on the left represents chromosome numbers and the red font on the right represents Wnt genes. 


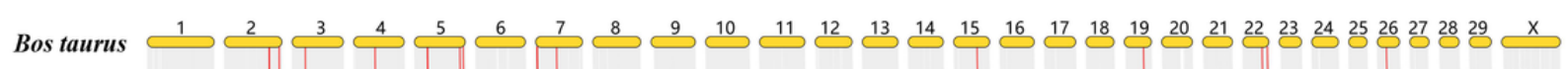

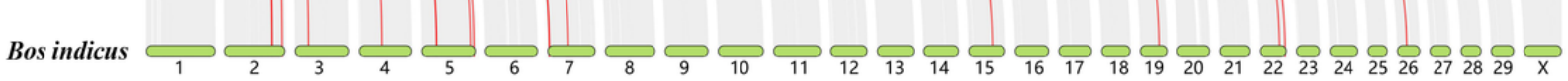

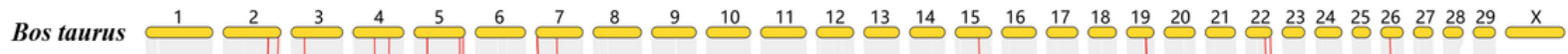

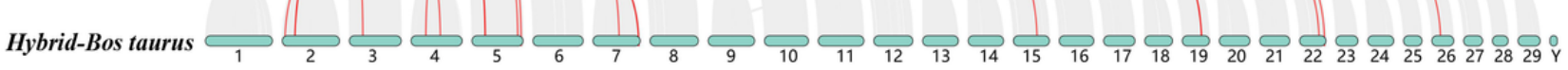

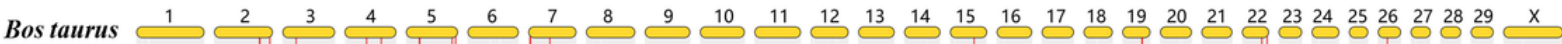
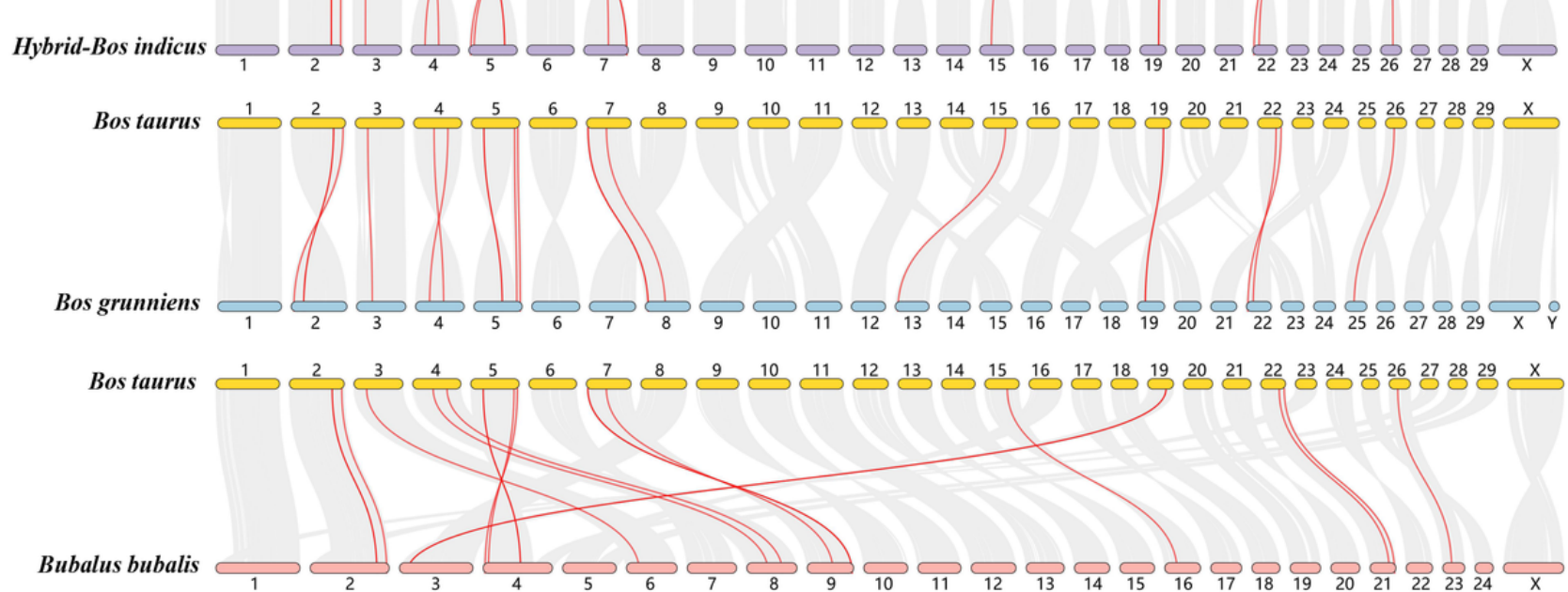

B
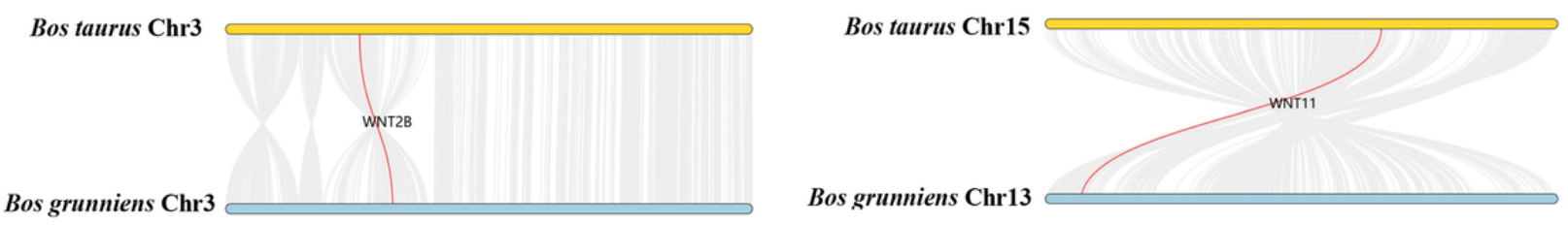

\section{Figure 4}

Collinearity analysis of Wnt genes between cattle and other organisms. Syntenic genes pairs are linked by grey lines whereas syntenic Wnt genes are shown as red lines. 
A

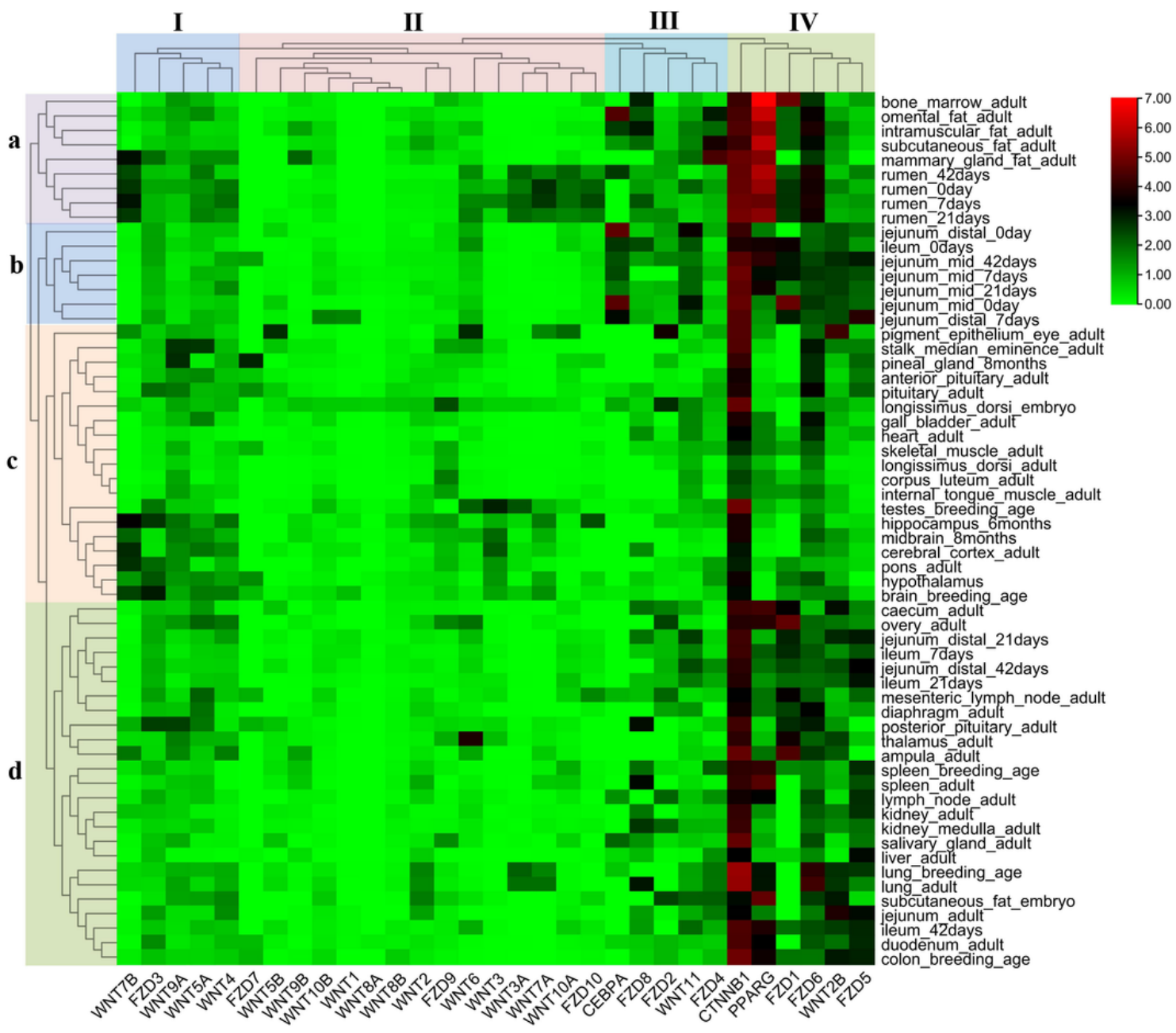

B

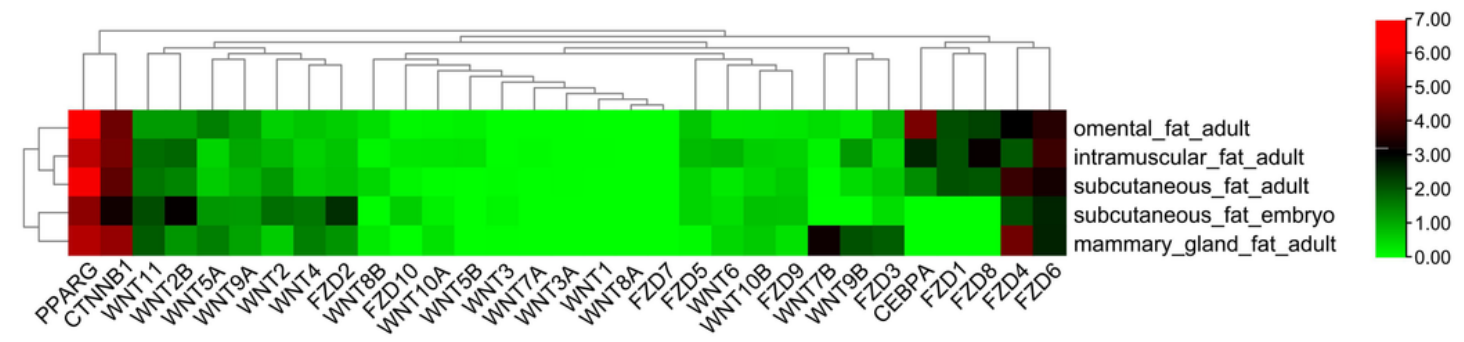

\section{Figure 5}

Expression analysis of the Wnt gene family in different bovine tissue types. A. Expression analysis of the Wnt gene family in 60

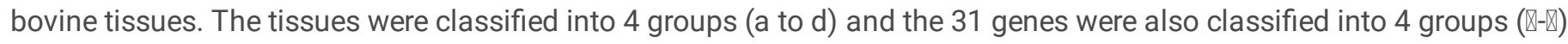
according to their expression pattern. B. Expression analysis of Wnt gene family in 5 bovine fat tissues. The horizontal axis represents genes and the vertical axis represents different bovine tissues. 
A

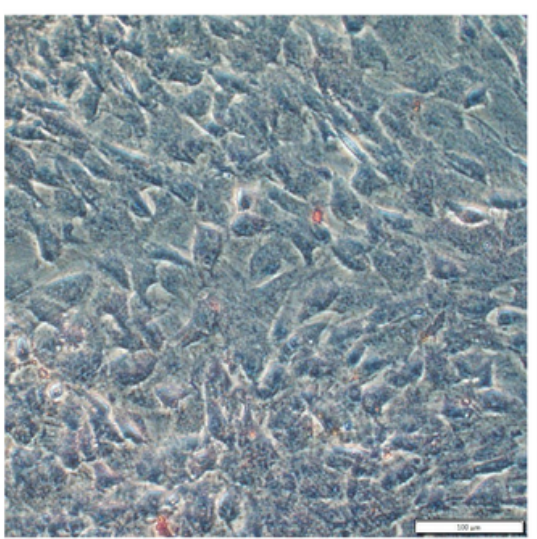

C

PPARY

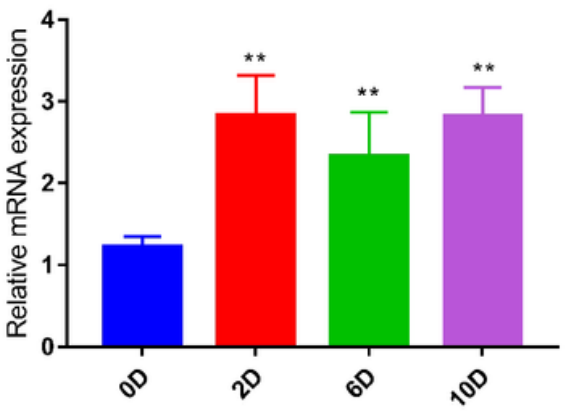

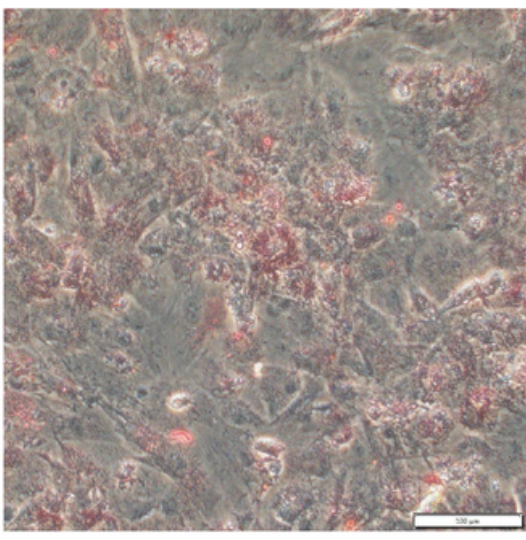

CEPBa
B

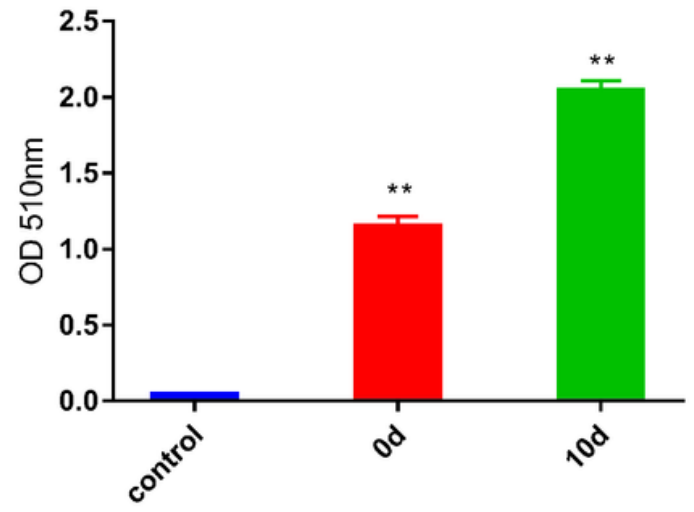

FABP4
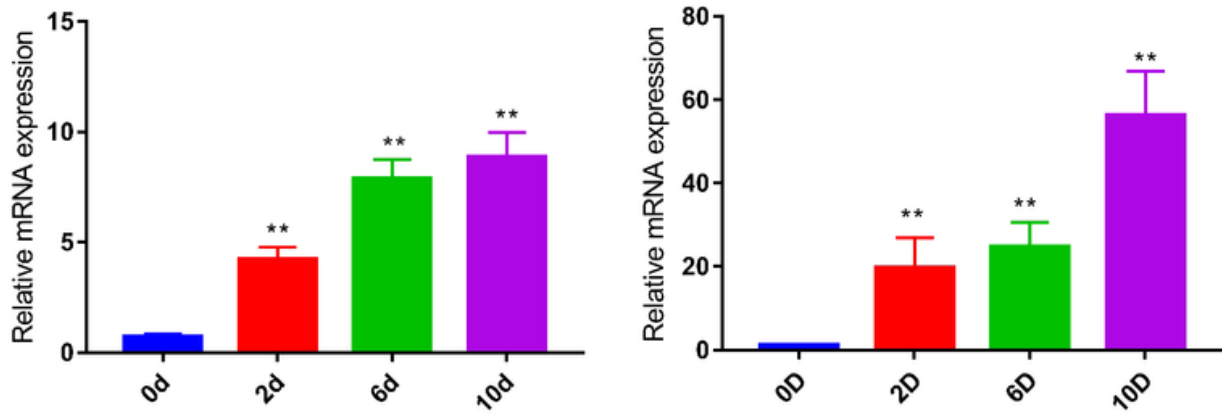

\section{Figure 6}

Induced differentiation of primary adipocytes. A. Oil Red 0 staining of bovine adipocytes induced at day 0 (left) and day 10 (right) of adipogenic diffferentiation. B. The measurements of absorbance at $510 \mathrm{~nm}$. Control: isopropanol, $0 \mathrm{~d}$ : substance extracted from adipocytes induced at 0 day, $10 \mathrm{~d}$ : substance extracted from adipocytes induced at 10 day. C. The expression of adipogenic marker genes during adipogenic diffferentiation. 
A
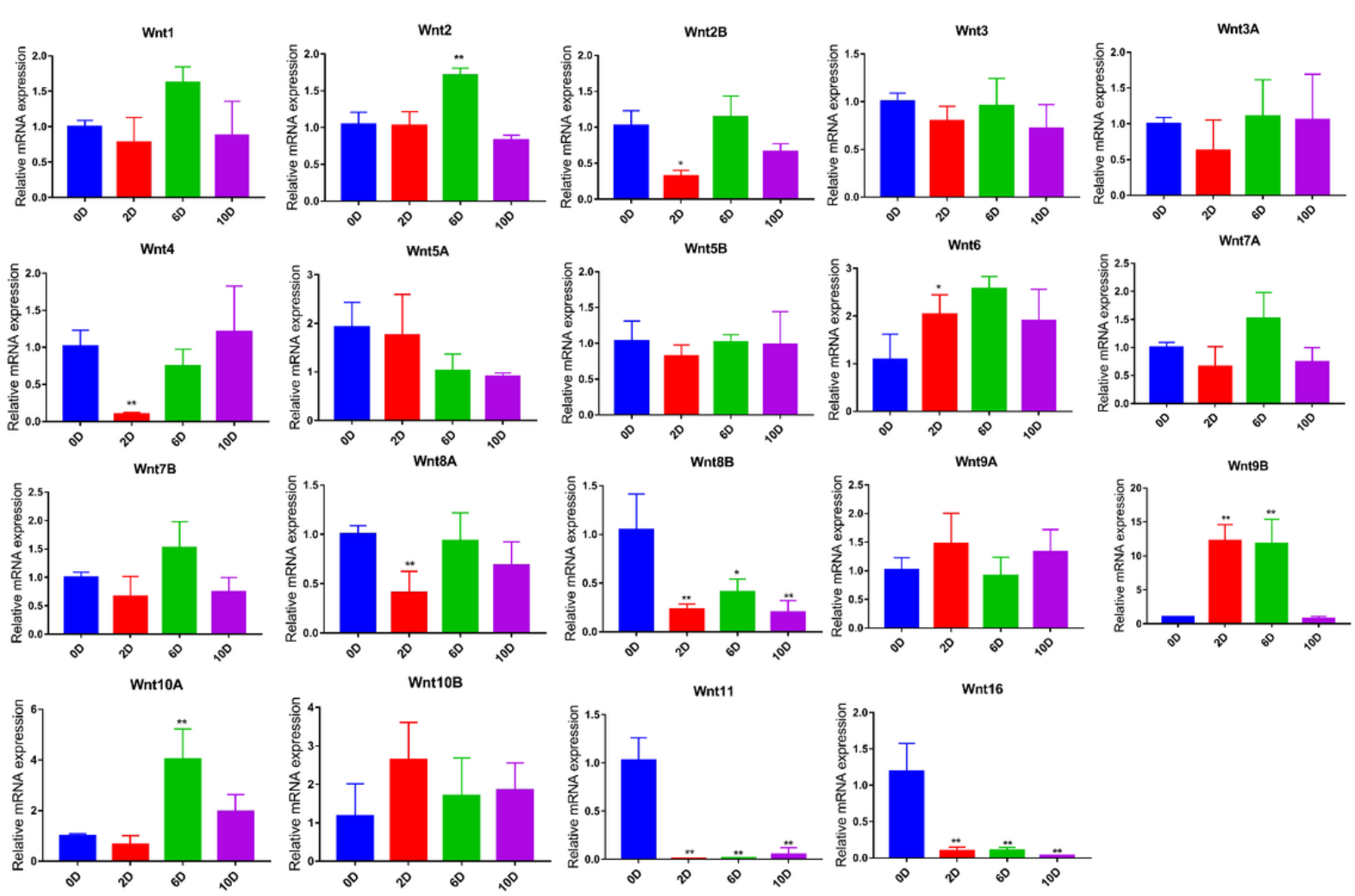

B
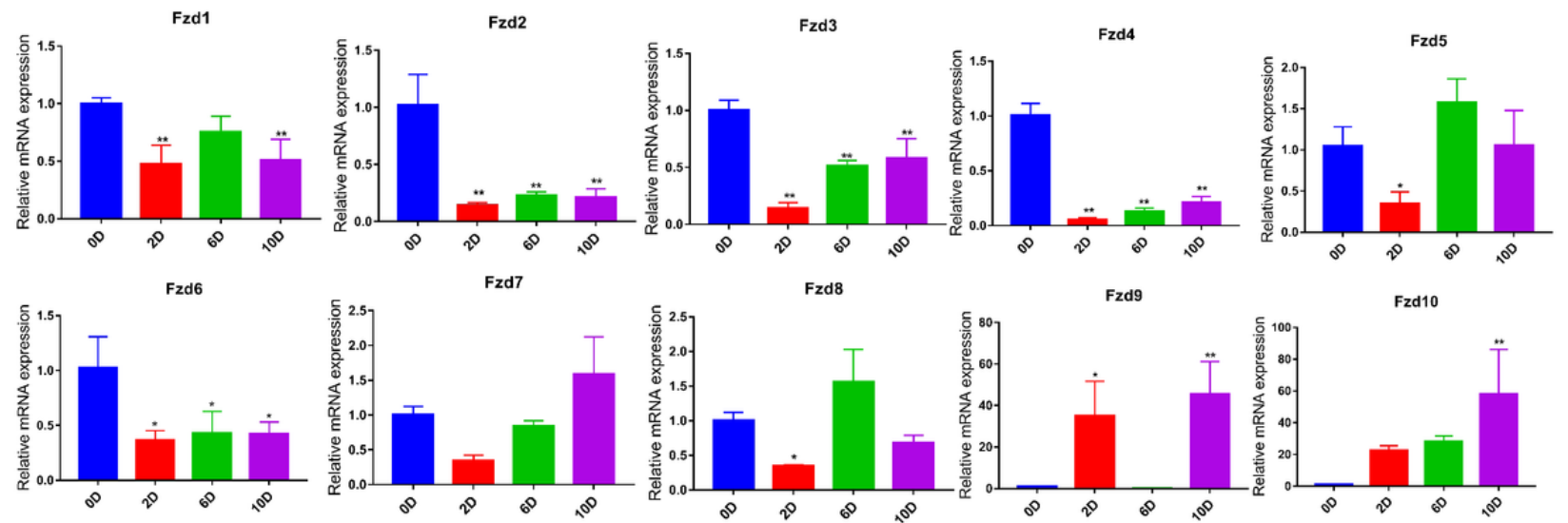

Figure 7

qPCR expression analysis of Wnt and its receptors Fzd family genes during adipocyte differentiation. A. The expression of 19 Wnt genes. B. The expression of $10 \mathrm{Fzd}$ genes. Error bars were gained from three measurements. Symbols * and ** above the bars indicate significant differences at a $p$ value of 0.05 and 0.01 respectively.

\section{Supplementary Files}

This is a list of supplementary files associated with this preprint. Click to download.

- Additionalfile1.xls

- Additionalfile2.txt

- Additionalfile3.pdf

- Additionalfile4.pdf

- Additionalfile5.pdf 
- Additionalfile6.pdf

- Additionalfile7.pdf

- Additionalfile8.xls.xls

- Additionalfile9.xls.xls

- AuthorChecklistFull.pdf

- srepchecklistforinitialsubmissions.pdf 